\title{
Performance Evaluation of Intelligent Adaptive Traffic Control Systems: A Case Study
}

\author{
Saeed Samadi ${ }^{1}$, Ali Pajoumand Rad ${ }^{2}$, Farhad Mohammad Kazemi ${ }^{3}$, Hamed Jafarian ${ }^{2}$ \\ ${ }^{1}$ Department of EE, Research Institute of Food Science \& Technology, Mashhad, Iran \\ ${ }^{2}$ Mashhad Traffic Control Center, Mashhad Traffic and Transportation Organization, Mashhad, Iran \\ ${ }^{3}$ Department of Computer Engineering, Payame Noor University, Mashhad, Iran \\ Email: s.samadi@rifst.ac.ir
}

Received April 12, 2012; revised May 13, 2012; accepted June 3, 2012

\begin{abstract}
Mashhad, the second largest city in Iran, like many other big cities, is faced with increasing traffic congestion owing to rapidly increasing population and annual pilgrimage. In recent years, Mashhad traffic and transportation authorities have been challenged with how to manage the increasing congestion with limited budgets for major roadway construction projects. Mashhad has recognized the need to improve the existing system capacity to get the most out of their current transportation system infrastructures. Since most of the delay times occur at signalized intersections, using an intelligent control system with proper capabilities to overcome the growing traffic requirements is recommended. Following comprehensive studies carried out with the aim of developing the Mashhad traffic control center, the SCATS adaptive traffic control system was introduced as the selected intelligent control system for integrating signalized intersections. The first intersection was equipped with this system in 2005. This paper describes the results of a field evaluation in which fixed actuated-coordinated signal timings are compared with those dynamically computed by SCATS. The effects of this system on optimizing fuel consumption as well as reducing air pollutants are fully discussed. It is found that SCATS consistently reduced travel times and the average delay per stopped or approaching vehicle. The positive impact of adaptive traffic control systems on fuel consumption and air pollution are also highlighted.
\end{abstract}

Keywords: ITS; Adaptive Traffic Control; SCATS; Measure of Effectiveness; Delay; Travel Time

\section{Introduction}

Intelligent Transportation Systems, or ITS, can be defined as the application of computing, information, and communications technologies to the real-time management of vehicles and networks involving the movement of people, goods, and services. When integrated into the transportation system's infrastructure, and into vehicles themselves, these technologies relieve congestion, improve safety, and enhance productivity. Intelligent Transportation Systems (ITS) encompasses a broad range of wireless and wire line communications-based information and electronics technologies [1].

Advanced Traffic Management System (ATMS) is a major subsystem of ITS, and real-time traffic control is considered to be one of the main operational processes of ATMS, with the aim of implementing adaptive traffic systems (Figure 1). The objects of this process are: 1) To minimize congestion while maximizing the movement of people and goods; 2) To improve the flow of traffic; and 3) To manage travel demand and prioritize it. The network of streets and roads plays an important role in urban traffic systems, and the quality of managing this network determines the success or failure of the whole system. Therefore, intersection control has its specific and important place as the most effective parameter influencing the quality of controlling street networks [2].

This paper describes the results of a field evaluation in which fixed actuated-coordinated signal timings are compared with those dynamically computed by the SCATS adaptive traffic control system. Travel times, travel time stopped, delays, and number of stops were collected by driving probe vehicles on the major selected routes, and information was extracted from traffic surveillance video cameras. Then, the main measures of effectiveness which are used in investigating the traffic condition of intersections are introduced, and the methodology of the study is also described. Finally, the results of studies carried out before and after using SCATS at selected intersections are fully analyzed.

\section{Signalized Intersections Control Systems}

\subsection{Pre-Time System}

It is the simplest method for controlling the traffic load 


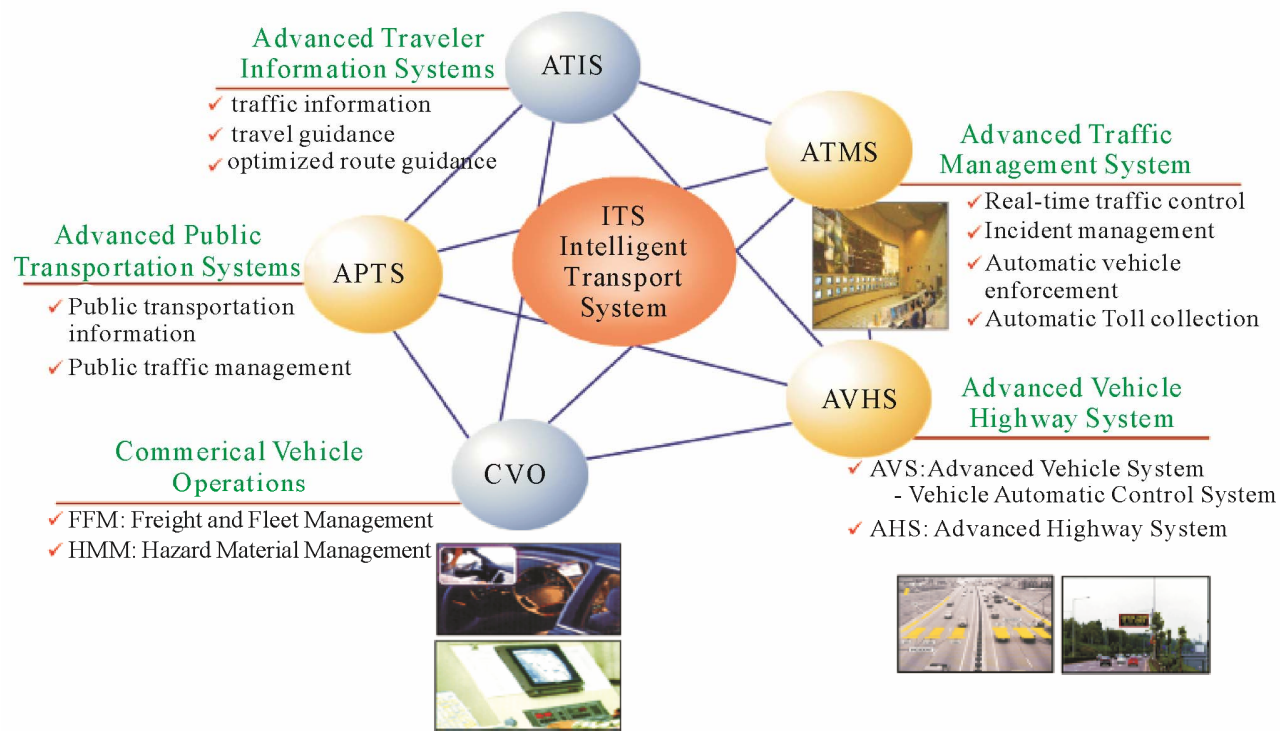

Figure 1. Outline of intelligent transportation systems.

of an intersection. In this system, regardless of traffic load, timing and phasing procedures are predetermined and recorded in the local memory of the system. The timing is set based on the statistics of the traffic load over different hours of a day and different days of a year. Obviously, this system lacks the capability of showing an appropriate response to traffic load variations. Especially when the traffic load does not follow a specific pattern, it cannot calculate and apply proper timing at intersections.

\subsection{Central Pre-Time System}

The next step in improving timing at intersections is to try to establish communication between intersections and the traffic control center. In this case, as all systems can communicate with a single control center, it is possible to change the timing and correct it via a single source (the traffic control center). Therefore, we can change the timing of intersections and improve their efficiency using the ITS system's peripheral equipment, like installed traffic cameras or the reports of supervisors.

\subsection{Central Intelligent System}

This is the newest signalized intersection control method in which the timing and phasing parameters are continuously adjusted to accommodate the real conditions of traffic. The system could be aware of the real traffic conditions via vehicle detectors installed in intersections. There are various types of detectors, like inductive loop, radar, or image detectors, of which the inductive detector is the cheapest one. As shown in Figure 2, inductive loop detectors consist of one simple coil whose induction magnitude varies as the number of vehicles in an intersection changes.

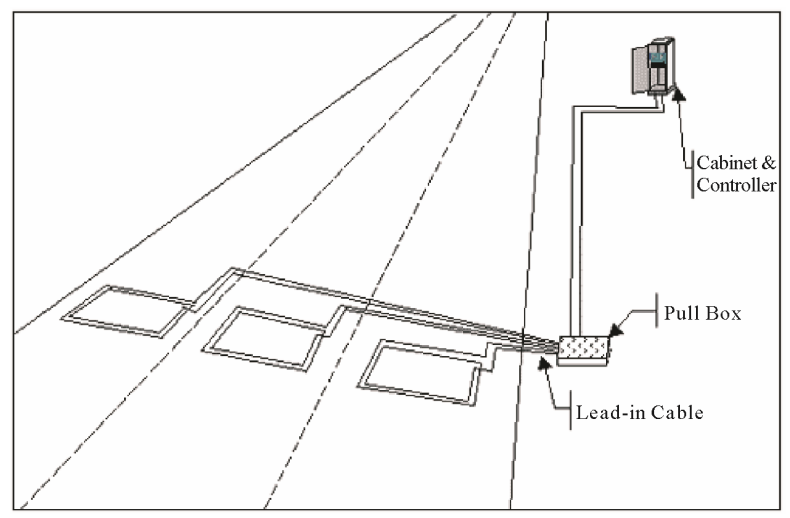

Figure 2. Inductive loop sensors.

The variations of induction are detected by the devices installed and are used for determining the traffic indexes of all four arms of an intersection. In this way, based on the instant information of a given intersection, the timing of each arm is calculated by central software and is applied immediately to the system in order to improve the system's efficiency. Figure 3 shows the differences between fixed-time and intelligent adaptive systems in controlling intersections at different hours of a day.

The system sends its different traffic information to central software. It is also able to optimally control the traffic arteries and to coordinate the next uninterrupted intersections. In this way, we can benefit from the green wave concept in intersections within a given route through decreasing the delay and stoppage times of vehicles in the route.

\section{SCATS}

Sydney Coordinated Adaptive Traffic System (SCATS) 
is a two-level, hierarchical traffic adaptive signal control system developed in Australia in the early 1980s by the Roads and Traffic Authority (RTA) [3]. SCATS uses information from vehicle detectors located in each lane immediately in advance of the stop line to adjust signal timings in response to variations in traffic demand and system capacity. SCATS acts as a heuristic feedback system, adjusting signal timings based on the changes in traffic flows during previous cycle(s). Two basic measures from detectors are used to adjust signal timings: degree of saturation (DS) and traffic flow. Both are measured each cycle. They serve to calculate cycle lengths, splits, and offsets for the following cycle. The SCATS strategy assumes that higher cycle lengths increase intersection capacity and splits proportional to approach demand, and provide longer offsets for increased traffic volumes. For saturated and over-saturated traffic conditions, SCATS usually abandons the concept of splits proportional to saturation and provides more green for higher traffic flows on major roads. For more information about SCATS logic, refer to the relevant literature [4-7].

As shown in Figure 4, SCATS can be deployed in the field with traffic signal controllers. A central computer

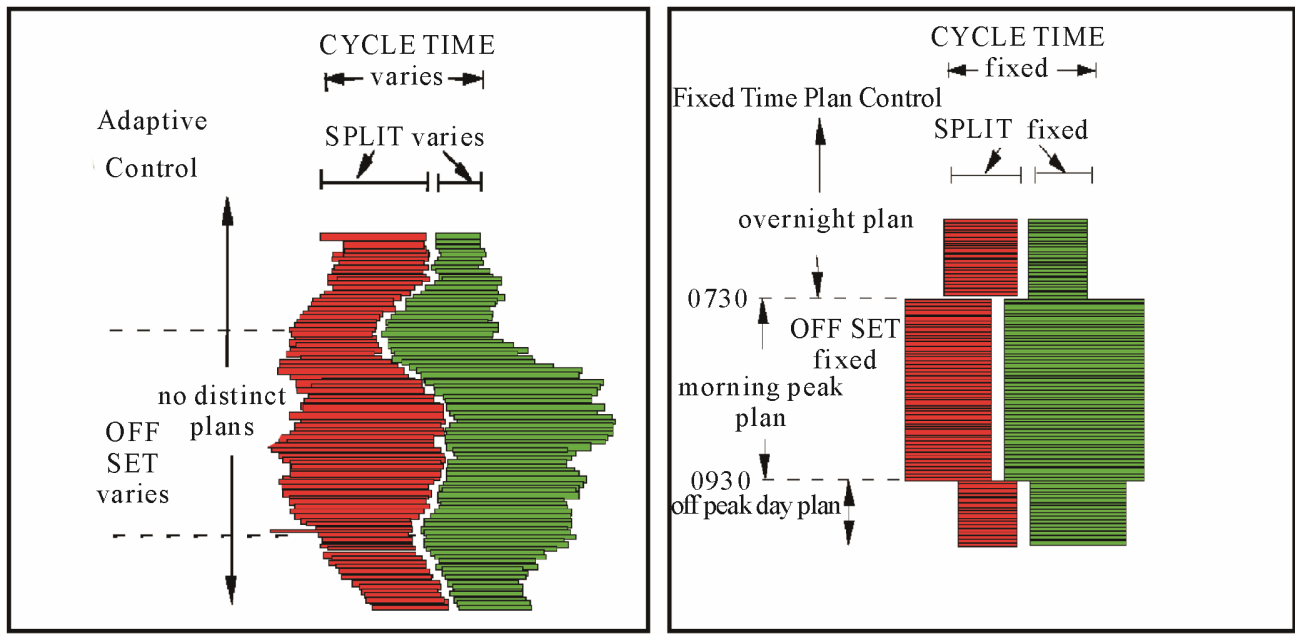

Figure 3. Comparison of the performance of fixed-time and intelligent (adaptive) systems.

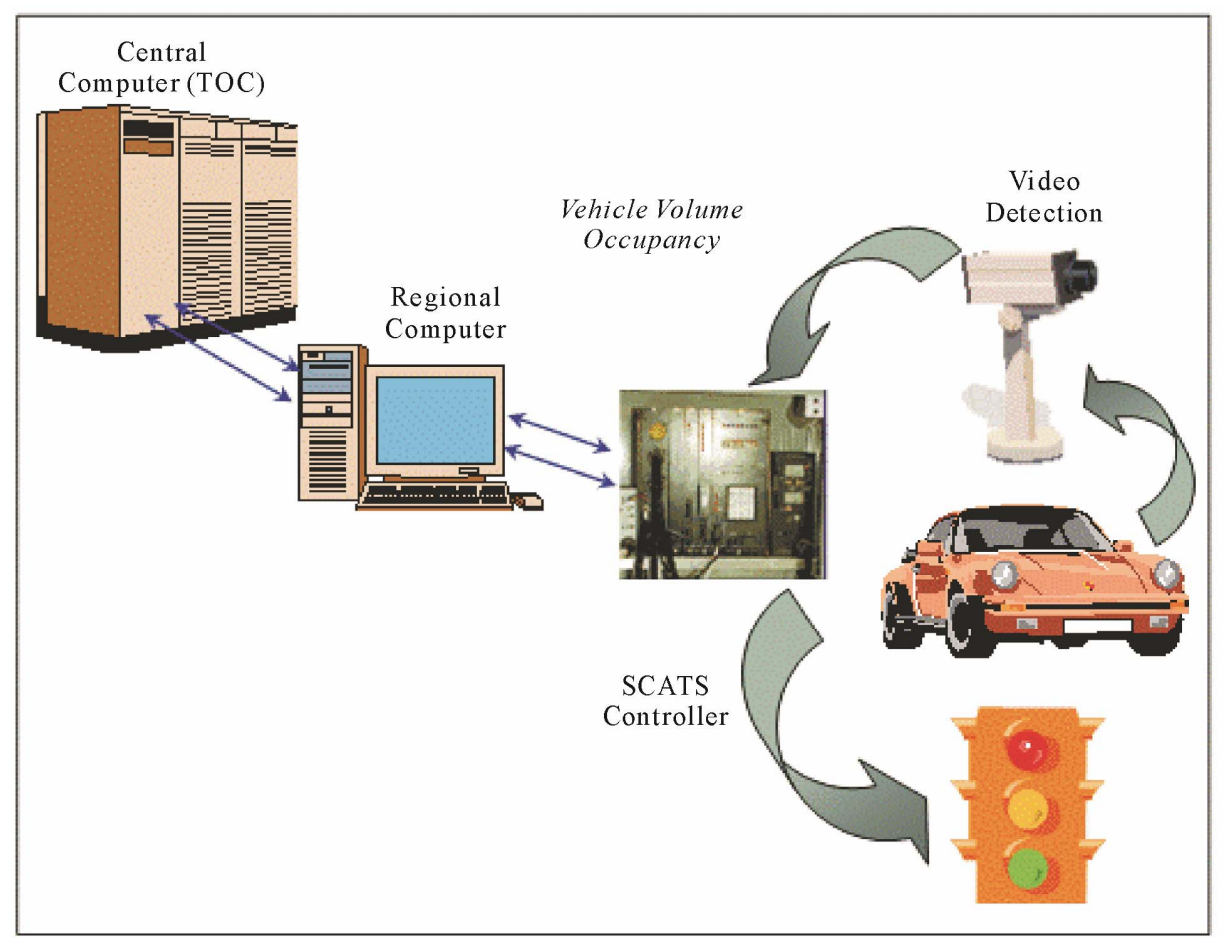

Figure 4. How SCATS works. 
running the SCATS algorithms processes DS and traffic flows from selected detectors in the system and adjusts signal timings in real time. The new signal timings are then sent to local controllers via the communication server and are implemented in the field. SCATS can deploy various levels of responsiveness when selecting the best signal timings (i.e. Masterlink, Flexilink, Isolated, Master Isolated and Fixed Time). If communication between the central and field components fails, pre-time signal timings from local controllers are implemented. Each regional computer can manage up to 250 intersections. A SCATS system can have up to 64 regional computers. Up to 100 users can connect to a SCATS central manager at the same time and up to 30 users can connect to a single regional computer simultaneously.

\section{Study Methodology}

In order to define the impacts of a system on improving traffic conditions in a given signalized intersection or in an initial street, we first should determine traffic measures of effectiveness (MOE) and then evaluate the impacts of the system on the defined measures.

In general, before and after studies are carried out with the aim of evaluating the quality of traffic flow within a given route, monitoring the variations of total travel and delay times, and determining major delay types. The outcomes of these studies are used to compare practical conditions of the route before and after improving an intersection or a route and optimizing the controlling parameters [8-12]. Usually these studies are done with the help of probe vehicles and statistical techniques.

According to the results of studies, total travel times within a given route as well as the average delay times of vehicles could be considered as two main parameters in determining the effectiveness of a given system or method in a signalized intersection. After computing these parameters in an intersection we can calculate other related parameters like the number of stops and the probable improvements in fuel consumption and air pollution levels.

Before calculating travel and delay times we should select the route that is going to be subjected to our studies as well as all controlling points. The most appropriate times for this study are: early morning, evening, and non-peak times of traffic in routes that are known to have the maximum traffic load. Case studies at other hours are also possible, if necessary.

The studies should be carried out in appropriate weather conditions in order to avoid the impacts of bad weather conditions. Since car accidents and unusually heavy traffic may result in unacceptable results, all travel that happens in these conditions should be eliminated. Once smooth traffic conditions have been reestablished, the study should be restarted. In summary, the studies should be carried out under usual and typical traffic conditions.

\subsection{Travel Time}

In order to measure travel times along the routes ending at the selected intersections, we use a probe vehicle that follows the traffic current. In this case, the vehicle should show a behavior similar to other vehicles, i.e., it should move or stop like other vehicles. This condition should be met in order to simulate the average motion of vehicles within a given route. In this method, two statistics men calculate the following parameters and record them in specific forms using two stopwatches as well as a distance-measuring device:

- Travel Time (TT) (seconds): the time within which the test vehicle travels from the start of the route to its end.

- Delay Time (DT): the time during which the vehicle has to stop because of route closure or reduce its speed below $10 \mathrm{~km} / \mathrm{hr}$.

- Running Time $(R T)$ (seconds): the total time consumed for the travel (regardless of delay times), which is derived from Equation (1):

$$
R T=T T-D
$$

- Running Speed $(R S)(\mathrm{km} / \mathrm{hr})$ : the average speed of the test vehicle during its motion (regardless of delay time), which is derived from Equation (2):

$$
R S=\text { Distance } / R T
$$

To calculate necessary movements for meeting the statistical requirements of before and after studies, we should run the following algorithm:

Estimate the count of go and back movements. After calculating the speeds of a group of movements, the real value of the difference between the first and the second movements, the second and the third and so on, is computed in order to define the exact count of movements. Then, the derived values are added together and the new obtained value is divided by the count of differences $(\mathrm{N}$ 1). The average range of movement speed will be derived through Equation (3) for initial information calculating purposes.

$$
R=S /(N-1)
$$

$R S=$ the mean of movement speed (miles per hour);

$R=$ the mean range of movement speed (miles per hour);

$S$ = the sum of the absolute magnitudes of differences;

$N=$ the number of required tests.

1) Again, define the necessary movements via Table 1. This time use the calculated average range.

2) Add other movements if necessary.

3) Consider experts' and engineers' points of view in 
order to adapt the count of movements with real conditions and eliminate non-logical values.

\subsection{Average Delay Time in an Intersection}

This measure is used to estimate the role of an intersection in facilitating traffic flow, i.e., it defines how vehicles arrive at the intersection and cross it or arrive at the intersection and change their lane. This index optimally gives detailed information about stoppage and delay times within all four arms of intersections. This study is based on counting the number of stopped vehicles in a given intersection during continuous and uninterrupted intervals. The mean time interval selected is 15 seconds; however, other intervals can be selected. The time intervals should be selected so that it does not result in multiple cycles of traffic lights. For example, if a traffic light has time cycles like 45, 60, 75, 90, 105, 120, 135 or 150 , we recommend 13 seconds as the time interval of study; otherwise, 15 seconds would be a proper time interval.

To start the study, the first statistics man counts and records the number of stopped vehicles within each interval. We can use a stopwatch to let him know the end of the interval. When a vehicle stops in the intersection for more than one interval, we should consider more than one delay time for it. In other words, a vehicle that has stopped in the intersection during successive intervals is counted in each of the intervals.

The second statistics man has a separate form and records the number and volume of the vehicles in the intersection. He classifies them to the stopped and moving categories within one interval. If we add the stoppage time of vehicles continually and count all stopped and moving vehicles, this will result in a considerable delay time. In case the study is carried out at an intersection equipped with traffic lights, the number of the vehicles which are going to stop (behind the stopped vehicles column) should consist of fully stopped vehicles. The vehicles that enter the intersection and cross it without stopping should be recorded in the non-stopped or mov- ing vehicles column.

The object of this study is to measure indexes like total delay time, mean delay time for each non-stopped vehicle, mean delay time for moving vehicles, and the percentage of stopped vehicles.

\section{Study Results}

At the end of this study, those intersections of Mashhad city equipped with the intelligent adaptive system were selected as our study locations (Figure 5). In order to investigate the impacts of this system more effectively, we tried to select roads on which some of their intersections have been equipped with SCATS system. Finally, the selected roads and intersections were studied in two main sections as follows:

- Fixed Time-Pre-Time versus SCATS control;

- Coordinated versus Local control.

The first section was carried out in the intersections equipped with the adaptive system, and we investigated the differences between fixed time and the SCATS system in parameters like travel time, delay time in intersecttions, and so on. These differences were calculated and reported in three intervals, i.e., early morning, end of night, and non-peak times of traffic. The second section of the study was carried out in the intersections equipped with an adaptive system with the aim of investigating the effects of central and coordinated control in adjacent intersections.

The results obtained were reported for three time intervals, i.e., early morning, end of night, and non-peak. Table 2 lists the selected intersections. The study was carried out as follows:

At first we studied the selected intersections, and the rate of vehicles crossing them at different times of a day was determined through the data extracted from the SCATS system. Then, the peak and non-peak times of the intersections were determined using the extracted data, and the technical study phase was started with respect to all necessary considerations. Prior to gathering statistical

Table 1. The approximate count and the minimum travels required for travel time study with a reliability of $>95 \%$.

\begin{tabular}{|c|c|c|c|c|c|}
\hline \multirow{2}{*}{ The average range of movement speedR ${ }^{\mathrm{a}}(\mathrm{mph})$} & \multicolumn{5}{|c|}{ The minimum count of movements versus permissible error } \\
\hline & $\pm 1.0 \mathrm{mph}$ & $\pm 2.0 \mathrm{mph}$ & $\pm 3.0 \mathrm{mph}$ & $\pm 4.0 \mathrm{mph}$ & $\pm 5.0 \mathrm{mph}$ \\
\hline 2.5 & 4 & 22 & 2 & 2 & 2 \\
\hline 5.0 & 8 & 4 & 3 & 2 & 2 \\
\hline 10.0 & 21 & 8 & 5 & 4 & 3 \\
\hline 15.0 & 38 & 14 & 8 & 6 & 5 \\
\hline 20.0 & 59 & 21 & 12 & 8 & 6 \\
\hline
\end{tabular}

${ }^{\mathrm{a}}$ If the value of $\mathrm{R}$ has not been mentioned above, recalculate it by interpolation. 


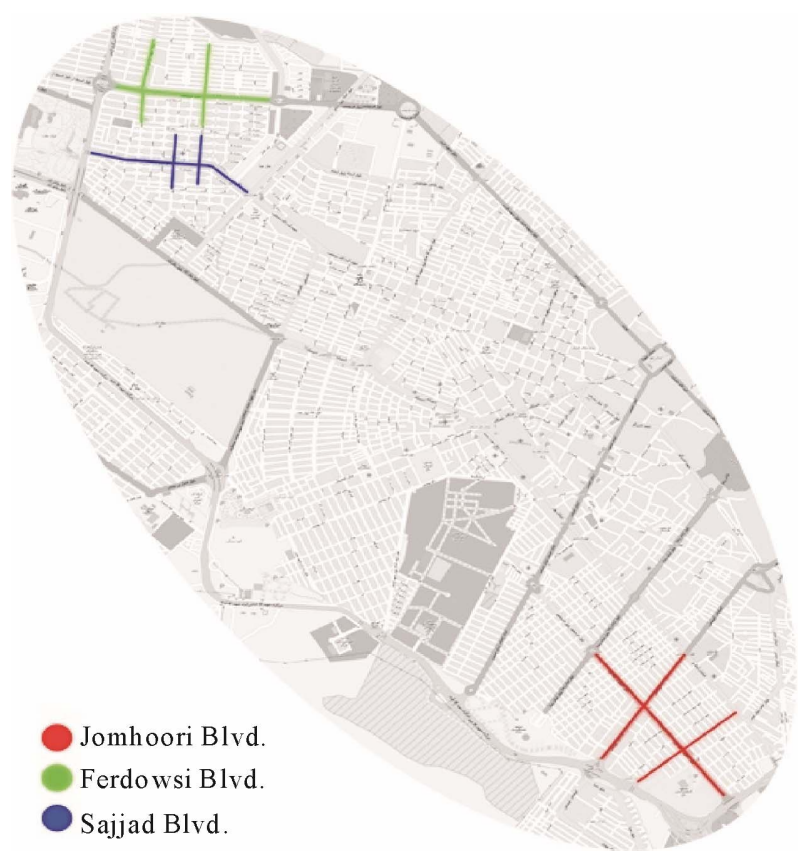

Figure 5. Selected routes on Mashhad map.

data from the selected routes, periodic inspections were carried out along these routes, and traffic load conditions were determined in those periods. Finally, the values of travel and delay times were calculated at early morning, end of night, afternoon, and non-peak times.

Table 3 shows the summary results of the study carried out on travel and delay times in three main roads consisting of six intersections. According to the table, the SCATS adaptive system shows evident improvements. Also, in Figure 6 comparative charts are shown of delay parameters for individual intersections and for all paths on average. After measuring the decreased rate of travel and delay times along each route as well as the parameters of movement components using the recorded information, we calculated fuel consumption and pollutant emission rates as well as improvement percentage in each route. Table 4 shows the results. As seen in this table, fuel consumption and $\mathrm{CO}$ and $\mathrm{HC}$ pollutant emissions were improved considerably so that for each vehi- cle per one kilometer, the fuel consumption rate decreased by 22, 15, and 47 milliliters respectively in Sajjad Boulevard, Ferdowsi Boulevard, and Jomhoorie-Eslami Boulevard.

Regarding the number of vehicles crossing the intersections during one day, we can calculate the daily optimized rate of fuel consumption [13]. For example, the total number of vehicles crossing Ferdowsi Boulevard during 24 hours of a day is estimated around 85,000. Therefore, we can calculate the optimized rate of fuel consumption as follows:

Daily optimization rate of fuel consumption in Ferdowsi Boulevard $=$ the average of the optimized rates of the fuel consumption of vehicles $\times$ the total vehicles crossing this route within 24 hours $=85,000$ (vehicle/day) $\times 15$ (mlit/vehicle) = 1275 (lit/day) .

On the other hand, if we extend this argument to the total number of vehicles crossing the mentioned intersections, we see a reduction in fuel consumption of 1200 , 1300, and 2900 lit/day respectively in Sajjad Boulevard, Ferdowsi Boulevard, and Jomhoori Boulevard. Moreover, as shown in Figure 7, the amount of the produced floating particles, i.e., CO and UHC (unburned hydrocarbons), are considerably decreased.

Finally, we can conclude that parameters like the quality of a smooth traffic flow, vehicles' speed increase, and delay time decrease, are all effective in significantly improving air pollution and gasoline consumption. There is just one negative parameter, i.e., the increase of $\mathrm{NO}_{\mathrm{x}}$ emissions, which increases as the vehicles' speed increases, though it has a less negative impact on the environment. As we know, the production rate of $\mathrm{NO}_{\mathrm{x}}$ pollutant is higher in CNG fuels compared to gasoline [14]; however, owing to the decrease of other factors, including fuel consumption and CO pollutant, our government is serious about using this fuel.

\section{Performance Improvement Suggestions}

On many roads of Mashhad city, traffic problems are generated from a few common sources. Since most routes suffer common problems, we can introduce general

Table 2. Selected intersections and their study objects.

\begin{tabular}{clll}
\hline Item & The Selected Route & Route Name & Study Object \\
\hline 1 & Jomhoori Blvd. & Jomhoori-Parvin & $\begin{array}{l}\text { Determining the optimizing rate of SCATS system compared } \\
\text { with pre-time case, before and after installation studies }\end{array}$ \\
3 & Ferdowsi Blvd. & Ferdowsi-Bahar & Determining the effectiveness of optimizing SCATS parameters and \\
4 & & Ferdowsi-Faramarz & investigating the green wave concept implemented in Ferdowsi Boulevard \\
5 & Sajjad Blvd. & Bozorgmehr-Sajjad & $\begin{array}{l}\text { Determining the optimizing rate of SCATS system compared with } \\
\text { pre-time case, before and after installation studies }\end{array}$ \\
\hline
\end{tabular}


Table 3. Summary results; Comparison table of delay parameters for all intersections times of traffic.

\begin{tabular}{|c|c|c|c|c|c|c|c|c|c|}
\hline \multirow{2}{*}{$\begin{array}{c}\text { Average \& Delay } \\
\text { Parameters/Time of Day }\end{array}$} & \multicolumn{3}{|c|}{ Morning Peak } & \multicolumn{3}{|c|}{ Evening Peak } & \multicolumn{3}{|c|}{ Normal Noon } \\
\hline & SCATS off & SCATS on & Changes (\%) & SCATS off & SCATS on & Changes (\%) & SCATS off & $\begin{array}{c}\text { SCATS } \\
\text { on }\end{array}$ & Changes (\%) \\
\hline \multicolumn{10}{|c|}{ Jomhoori Blvd. } \\
\hline $\begin{array}{l}\text { Average Delay Per } \\
\text { Stopped Vehicle }\end{array}$ & 33.3 & 31.0 & -6.9 & 33.0 & 30.0 & -9.0 & 28.1 & 25.1 & -10.7 \\
\hline $\begin{array}{l}\text { Average Delay Per } \\
\text { Approach Vehicle }\end{array}$ & 22.5 & 19.1 & -15.1 & 22.3 & 18.8 & -15.9 & 17.2 & 14.2 & -17.4 \\
\hline $\begin{array}{l}\text { Average Travel Time } \\
\text { of East to West Path }\end{array}$ & 179.1 & 170.0 & -5.1 & 186.0 & 176.6 & -5.1 & 154.8 & 143.5 & -7.3 \\
\hline $\begin{array}{l}\text { Average Travel Time } \\
\text { of West to East Path }\end{array}$ & 202.1 & 145.0 & -28.3 & 190.1 & 179.6 & -5.5 & 134.1 & 131.8 & -1.7 \\
\hline \multicolumn{10}{|c|}{ Ferdowsi Blvd. } \\
\hline $\begin{array}{l}\text { Average Delay Per } \\
\text { Stopped Vehicle }\end{array}$ & 33.0 & 31.5 & -4.5 & 34.9 & 33.0 & -5.4 & 32.5 & 30.9 & -4.9 \\
\hline $\begin{array}{l}\text { Average Delay Per } \\
\text { Approach Vehicle }\end{array}$ & 22.1 & 20.7 & -6.3 & 22.7 & 19.0 & -16.3 & 20.8 & 19.1 & -8.2 \\
\hline $\begin{array}{l}\text { Average travel time } \\
\text { of East to West path }\end{array}$ & 138.1 & 125.8 & -8.9 & 143.3 & 139.5 & -2.7 & 125.5 & 123.7 & -1.4 \\
\hline $\begin{array}{l}\text { Average travel time } \\
\text { of West to East path }\end{array}$ & 143.6 & 141.5 & -1.5 & 144.0 & 119.5 & -17.0 & 128.1 & 122.3 & -4.5 \\
\hline \multicolumn{10}{|c|}{ Sajjad Blvd. } \\
\hline $\begin{array}{l}\text { Average Delay Per } \\
\text { Stopped Vehicle }\end{array}$ & 34.3 & 31.1 & -9.3 & 33.6 & 30.4 & -9.5 & 29.9 & 27.6 & -7.7 \\
\hline $\begin{array}{l}\text { Average Delay Per } \\
\text { Approach Vehicle }\end{array}$ & 25.6 & 23.4 & -8.6 & 25.8 & 23.0 & -10.9 & 20.0 & 16.3 & -18.5 \\
\hline $\begin{array}{l}\text { Average Travel Time } \\
\text { of East to West Path }\end{array}$ & 192.3 & 167.8 & -12.7 & 319.6 & 282.5 & -11.6 & 87.3 & 87.8 & 0.6 \\
\hline $\begin{array}{l}\text { Average Travel Time } \\
\text { of West to East Path }\end{array}$ & 143.5 & 137.0 & -4.5 & 192.1 & 160.5 & -16.4 & 100.6 & 70.0 & -30.4 \\
\hline \multicolumn{10}{|c|}{ Average Parameters for All Routes } \\
\hline $\begin{array}{l}\text { Average Delay Per } \\
\text { Stopped Vehicle }\end{array}$ & 33.5 & 31.2 & -7.0 & 33.8 & 31.1 & -7.9 & 30.2 & 27.9 & -7.6 \\
\hline $\begin{array}{l}\text { Average Delay Per } \\
\text { Approach Vehicle }\end{array}$ & 23.4 & 21.1 & -10.0 & 23.6 & 20.3 & -14.2 & 19.3 & 16.5 & -14.5 \\
\hline $\begin{array}{l}\text { Average Travel Time } \\
\text { of East to West Path }\end{array}$ & 169.8 & 154.5 & -9.0 & 216.3 & 199.5 & -7.8 & 122.5 & 118.3 & -3.4 \\
\hline $\begin{array}{l}\text { Average Travel Time } \\
\text { of West to East Path }\end{array}$ & 163.1 & 141.2 & -13.4 & 175.4 & 153.2 & -12.7 & 120.9 & 108.0 & -10.7 \\
\hline
\end{tabular}

Note: The unit of "the mean of travel times sum", "the mean of delay times sum" and "the mean of run times sum" indexes is seconds. The unit of "the mean of travel speed sum" and "the mean of run speed sum” is $\mathrm{km} / \mathrm{hr}$ (kilometer per hour).

solutions for removing similar traffic knots. Based on the results of field studies and observations, previous sections introduced separate solutions for removing the traffic problems of each route. In this section, we suggest some general solutions enabling optimize use of the SCATS central adaptive control system as follows:

\subsection{The Geometrical Parameters of Signalized Intersections Should Be Corrected}

Since in intersections whose geometrical dimensions have been corrected vehicles move in canalized right turn, straight forward, and left turn routes, they will undoubtedly encounter each other less. Thus, in these intersections the adaptive system can play a significant role in improving traffic indexes through applying optimized timing on the arms of the intersections. This correction has another positive impact, which is the canalization of pedestrian routes so that by assuming that they pay attention to traffic lights, which has been considered in SCATS system, this can decrease convergence between vehicles and pedestrians, which in turn will cause the intersections to be evacuated more quickly.

\subsection{The Phasing of Intersections Should Be Corrected}

In signalized intersections the convergence of right turn and straightforward movements reduces the speed of motion and increases delay times. Since the SCATS system is equipped with appropriate sensors at the entrance of intersections, it is capable of identifying traffic problems by analyzing the effects of the generated traffic knot on the flow of intersections, and, obviously, it will correct 

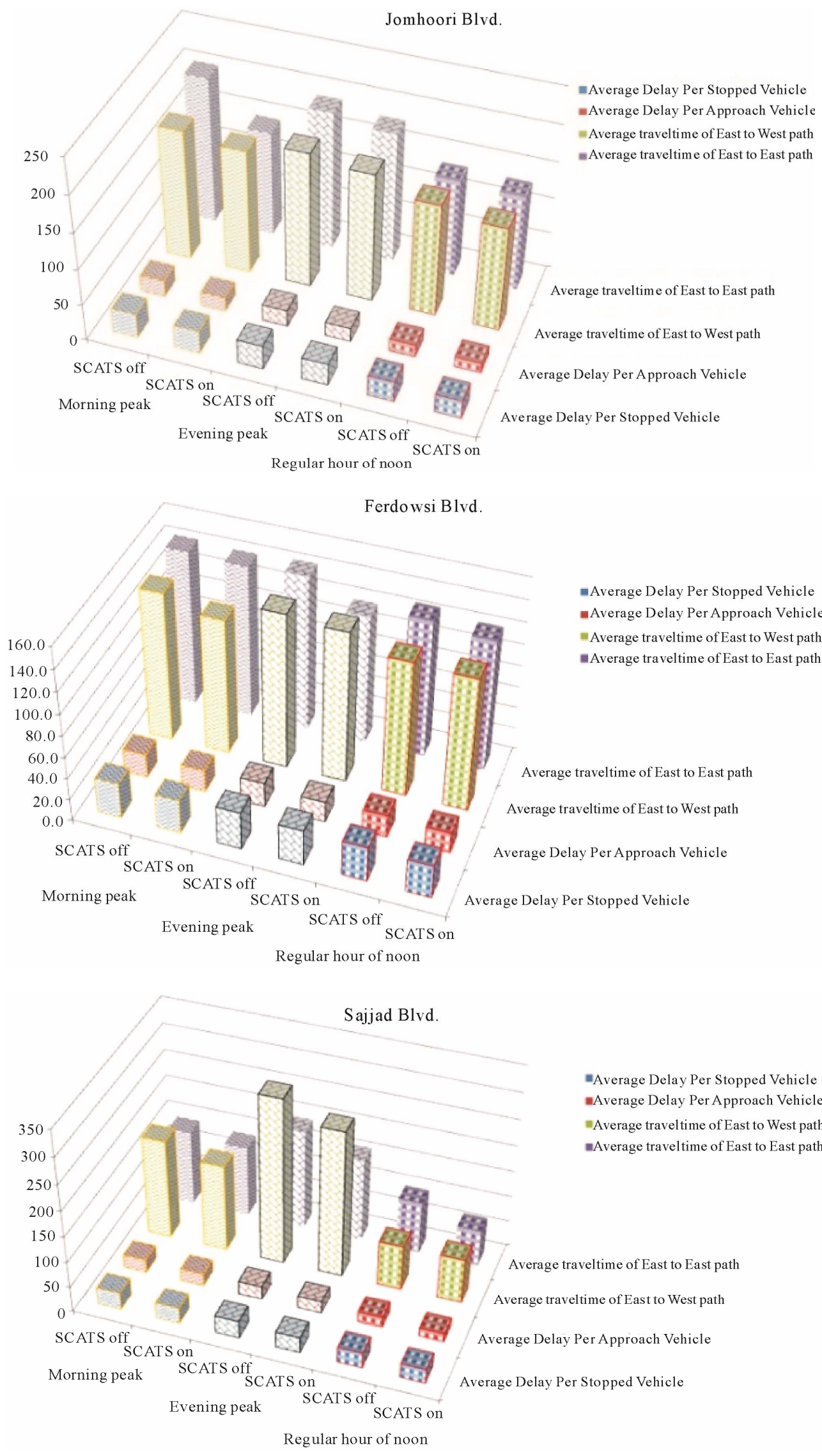


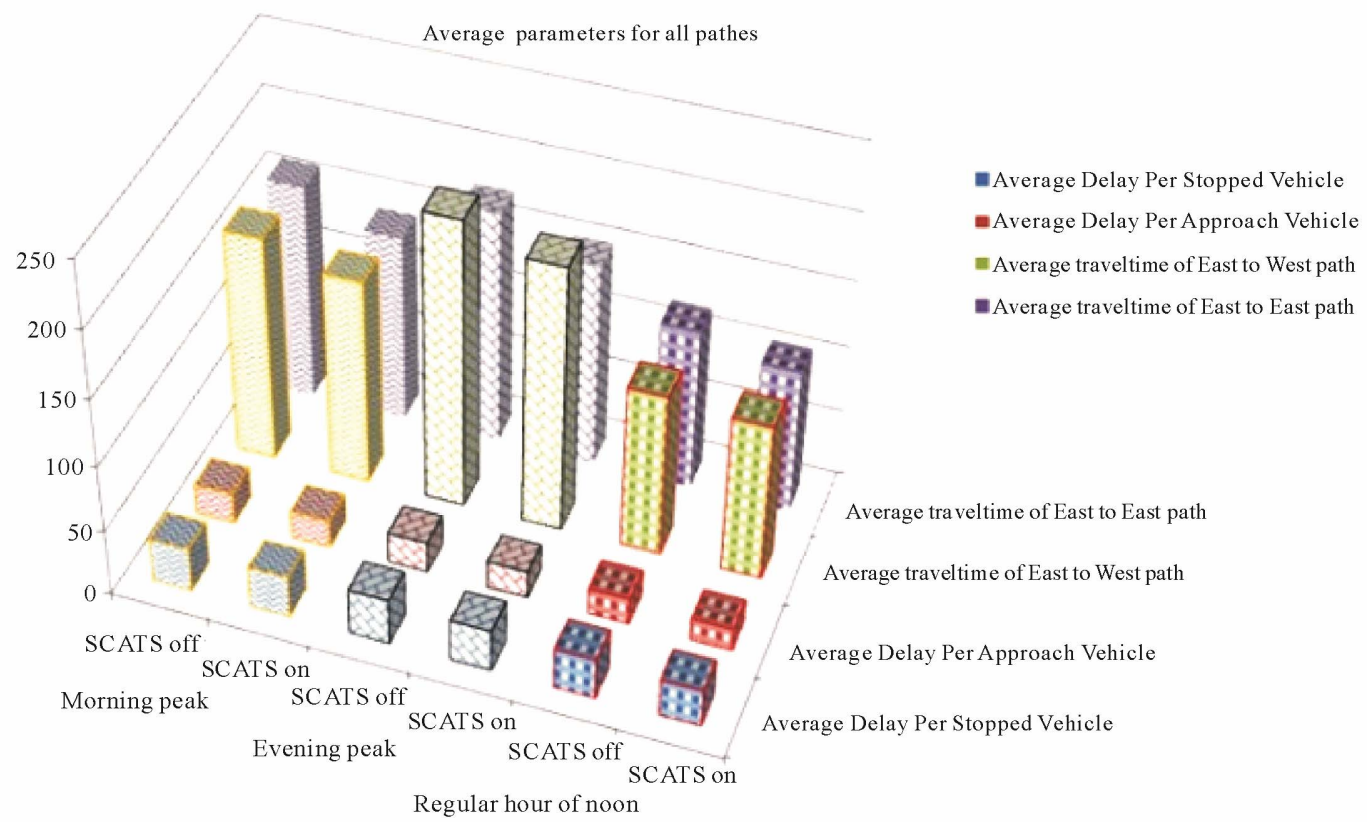

Figure 6. Comparative charts of delay parameters for all intersections.

Table 4. The impacts of SCATS on fuel consumption and air pollution.

\begin{tabular}{|c|c|c|c|c|c|c|c|c|c|}
\hline Location & Time & Condition & $\begin{array}{c}\text { Fuel } \\
\text { Consumption } \\
\text { (Lit) }\end{array}$ & $\begin{array}{c}\text { CO Emissions } \\
\text { (gr) }\end{array}$ & $\begin{array}{c}\mathrm{HC}_{\mathrm{X}} \text { Emissions } \\
\text { (gr) }\end{array}$ & $\begin{array}{c}\text { NO Xemissions } \\
\text { (gr) }\end{array}$ & $\begin{array}{c}\text { Fuel } \\
\text { Consumption } \\
\text { Decrease (\%) }\end{array}$ & $\begin{array}{c}\text { CO } \\
\text { Emissions } \\
\text { Decrease (\%) }\end{array}$ & $\begin{array}{r}\text { HC Emissions } \\
\text { Decrease (\%) }\end{array}$ \\
\hline \multirow[t]{6}{*}{$\begin{array}{l}\text { Ferdowsi } \\
\text { Blvd. }\end{array}$} & Morning Peak & Before & 0.404 & 55.689 & 4.953 & 1.879 & 3.7 & 6.2 & 5.9 \\
\hline & & After & 0.389 & 52.256 & 4.661 & 1.988 & & & \\
\hline & Evening Peak & Before & 0.412 & 56.652 & 5.026 & 1.841 & 5.2 & 11 & 10.3 \\
\hline & & After & 0.39 & 50.445 & 4.51 & 2.06 & & & \\
\hline & Normalnoon & Before & 0.387 & 48.678 & 4.344 & 2.082 & 5.6 & 4.4 & 4.5 \\
\hline & & After & 0.366 & 46.524 & 4.146 & 2.143 & & & \\
\hline \multirow[t]{6}{*}{$\begin{array}{l}\text { Jomhoori } \\
\text { Blvd. }\end{array}$} & Morning Peak & Before & 0.503 & 73.059 & 6.396 & 2.194 & 9.2 & 17.2 & 15.8 \\
\hline & & After & 0.456 & 60.519 & 5.383 & 2.653 & & & \\
\hline & Evening Peak & Before & 0.498 & 71.971 & 6.304 & 2.213 & 4.2 & 6.1 & 6 \\
\hline & & After & 0.477 & 67.57 & 5.927 & 2.323 & & & \\
\hline & Normal Noon & Before & 0.439 & 57.741 & 5.127 & 2.697 & 6.6 & 12.8 & 11.7 \\
\hline & & After & 0.41 & 50.328 & 4.53 & 3.106 & & & \\
\hline \multirow[t]{6}{*}{$\begin{array}{l}\text { Sajjad } \\
\text { Blvd. }\end{array}$} & Morning Peak & Before & 0.37 & 60.917 & 5.328 & 0.796 & 5.8 & 6.5 & 6.7 \\
\hline & & After & 0.348 & 56.938 & 4.972 & 0.803 & & & \\
\hline & Evening Peak & Before & 0.432 & 78.794 & 6.786 & 0.755 & 15.9 & 21.9 & 21.3 \\
\hline & & After & 0.363 & 61.539 & 5.342 & 0.77 & & & \\
\hline & Normal Noon & Before & 0.286 & 39.49 & 3.552 & 1.07 & 8.8 & 15.9 & 14.5 \\
\hline & & After & 0.261 & 33.218 & 3.035 & 1.276 & & & \\
\hline
\end{tabular}


Ferdowsi Blvd

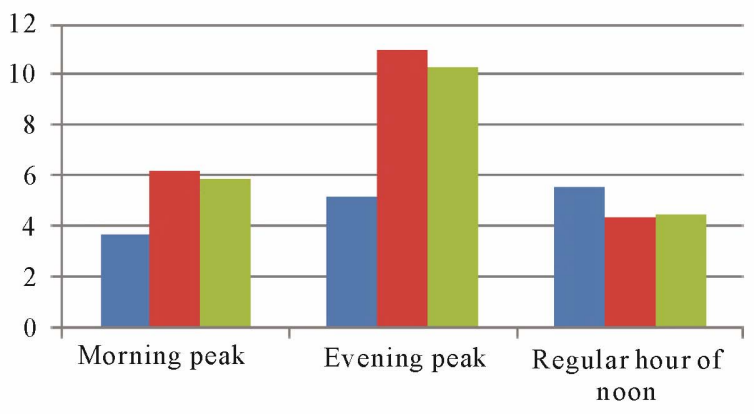

Jomhoori Blvd

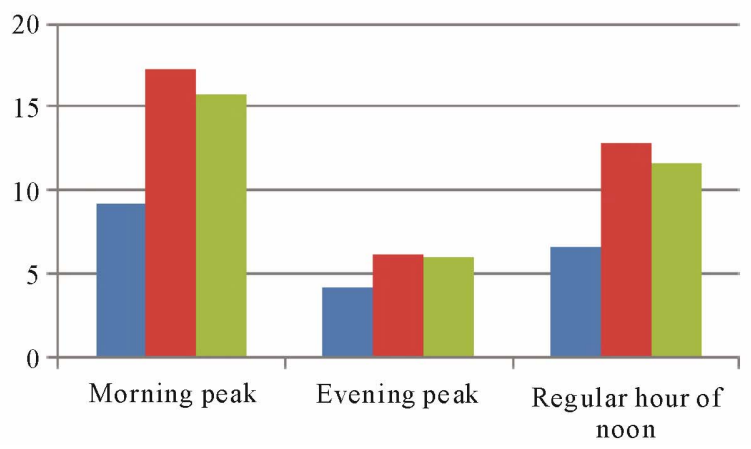

Sajjad Blvd

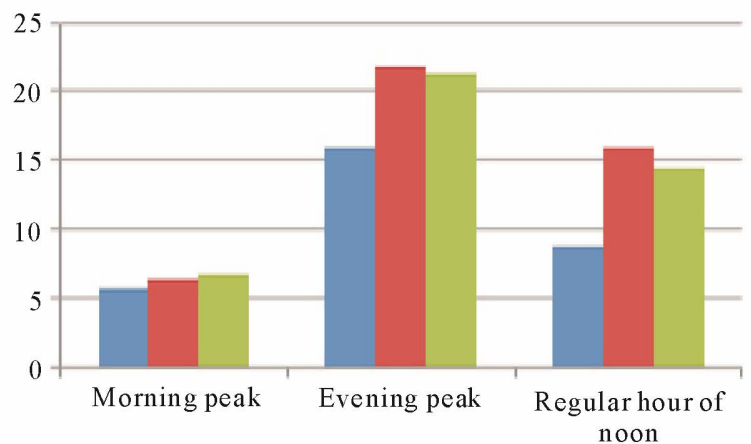

percent decrease in fuel consumed

percent decrease in $\mathrm{CO}$ emissions

percent decrease in $\mathrm{HC}$ emissions

percent decrease in fuel consumed

percent decrease in $\mathrm{CO}$ emissions

- percent decrease in $\mathrm{HC}$ emissions percent decrease in fuel consumed

percent decrease in $\mathrm{CO}$ emissions

- percent decrease in $\mathrm{HC}$ emissions

Figure 7. Comparison of fuel consumption and air pollution reduction at different times of day using SCATS.

timing in this condition. In the cases that left turn movements are dominant in an intersection, it is advised that the left turn movements be separated from straight forward ones if possible in order to establish a smooth traffic flow in this type of intersection. Of course, this separation will lead to the increasing of phase numbers, the red time duration of each phase, and probably the increasing of the delay time of each stopped vehicle. It will, however, eventually lead to a smoother traffic flow, because the use of the adaptive control system, which balances the timing of each phase with respect to the traffic load within each arm of intersection, will finally decrease travel and delay times. So, it is advised to determine the considerations in separate phases, provided that we have sufficient space and capacity and technical traffic considerations are being considered.

\subsection{Drivers and Pedestrians Should Have Respect for the Law}

The adaptive control of signalized intersections is only one parameter among several important parameters that are considered in optimizing the traffic conditions of routes. Respect for the traffic laws and the promotion of the traffic culture of drivers and pedestrians is one of the most effective parameters resulting in a smooth traffic flow and improvement of traffic indexes.

Here we enlist in summary the chief topics to be observed by citizens: 
- Drivers should select their appropriate lane before they arrive at intersections.

- They should observe pedestrians paths and drive in the specified phases for pedestrians with respect to traffic lights.

- They should not park their cars within the intersection's limits.

- Taxi cabs should not take on or let off passengers after and before the intersection's limits.

- They should not double park in drive bands.

- They should pay attention to traffic lights and should drive at safe speed limits.

- It is necessary to organize marginal auto parks and assign sufficient parking spaces outside signalized intersections and heavy traffic roads.

\subsection{The System Parameters Should Be Optimized Continuously}

SCATS is an intelligent system and adapts itself with traffic flow; however, determining all of its parameters requires presumptions that are determined by the system designer. Predefined factors should be reviewed continuously, such as the minimum and maximum timings, or the programs that set the proper cycle length, or the percentage of each phase in each route. It is possible to know the traffic conditions in previous hours and days as well as the volume of data extracted by induced sensors using peripheral software like the SCATS traffic reporter. This kind of software provides other valuable information like the saturation percentage of each route. These data, along with filed observations carried out with the aim of getting information from traffic officers, can make an appropriate base for optimizing the main parameters for intersection control purposes.

\subsection{Drivers Should Be Informed of Traffic Conditions}

Displaying the traffic conditions of signalized intersections using the information derived from induced sensors is one of the SCATS system's capabilities. This advantage makes it possible to know the traffic conditions of each arm of an intersection as well as the traffic conditions of the routes ending at those intersections. Therefore, we could use this advantage and inform drivers of the traffic conditions of routes and enable them to select optimum routes in order to avoid heavy traffic loads on specific routes. Drivers can be informed by the operator via news channels, through the Internet, and via displaying the traffic map of city, which is presented by the software.

\section{Conclusions}

To summarize the results of this before and after study, it is shown that in most selected routes and intersections the SCATS adaptive system has positive impacts on travel and delay times. However, the effectiveness of this system differs from route to route and depends on the conditions of a given route. In other words, at main routes with a high volume of traffic, lowering the delay and vehicle stoppage time has a higher priority for the intelligent traffic control system. Although, this may temporarily increase the delay in other routes, but regarding traffic volume of each route it will increase overall performance of the system. The obtained results, however, show that more attention should be paid to the quality of defining the parameters of the adaptive system in the design and maintenance phases.

It should be mentioned that this study has been carried out on routes with heavy traffic conditions. In this type of route, the intersection commanding system and its specified timing are not sufficient for reducing traffic load, and other parameters may play significant role for this purpose. An intersection adaptive control system, as an effective parameter, can significantly improve the traffic indexes of intersections, which in turn will improve the traffic conditions of routes, but this improvement does not solely depend on this parameter.

Some helpful suggestions for performance improvement of the system are also given at the end of the paper.

\section{Acknowledgements}

This evaluation was funded jointly by Mashhad Traffic and Transportation Organization and Iran Ministry of Science, Research \& Technology.

\section{REFERENCES}

[1] L. Figueiredo, et al., "Towards the Development of Intelligent Transportation Systems," IEEE Proceedings of Intelligent Transportation Systems, Oakland, 25-29 August 2001, pp. 1206-1211.

[2] N. Gartner, et al., "Development of Advanced Traffic Signal Control Strategies for Intelligent Transportation Systems: Multilevel Design,” Transportation Research Record, No 1494, 1995, pp. 98-105.

[3] P. R. Lowrie, "SCATS, Sydney Co-Ordinated Adaptive Traffic System: A Traffic Responsive Method of Controlling Urban Traffic,” Roads and Traffic Authority NSW, Darlinghurst, 1990. http://library.its.berkeley.edu/

[4] R. Akcelik, M. Besly and E. Chung, "An Evolution of SCATS Master Isolated Control," Proceedings of the 19th ARRB Transport Research Conference (Transport 98), 1998, pp. 1-24.

[5] C. J. Khisty, "Transportation Engineering: An Introduction,” 2nd editon, Prentice-Hall, Upper Saddle River, 1998.

[6] www.scats.com.au

[7] R. Ghaman, D. Gettman, L. Head and P. B. Mirchandani, "Adaptive Control Software for Distributed Systems," 
28th Annual Conference of the Industrial Electronics Society, 5-8 November 2002, pp. 3103-3106.

[8] H. D. Robertson and J. E. Hummer, "Manual of Transportation Engineering Studies,” Prentice-Hall, Upper Saddle River, 1994.

[9] K. Fehon and R. Chong, "Adaptive Traffic Signal System for Cupertino California,” 4th Asia-Pacific Transportation Development Conference, April 18-20, 2003.

[10] F. Zhu, G. Li, Zh. Li, C. Chen and D. Wen, “A Case Study of Evaluating Traffic Signal Control Systems Using Computational Experiments," IEEE Transaction on Intelligent Transportation Systems, Vol. 12, No. 4, 2011, pp. 1220-1226. doi:10.1109/TITS.2011.2157691

[11] F. Hu, et al., "Field Evaluation of SCATS Control System in Las Vegas," Proceedings of the 11th International Conference of Chinese Transportation Professionals, 2011, pp. 3963-3973. doi:10.1061/41186(421)397

[12] M. Kergaye, et al., "Comparison of before-after versus off-on Adaptive Traffic Control Evaluations: Park City,” Case Study, 2006.

[13] “MUTS: Manual on Uniform Traffic Studies,” 2000.

[14] P. F. Everall, "The Effects of Road and Traffic Conditions on Fuel Consumption,” Road Research Laboratory, 1983. 